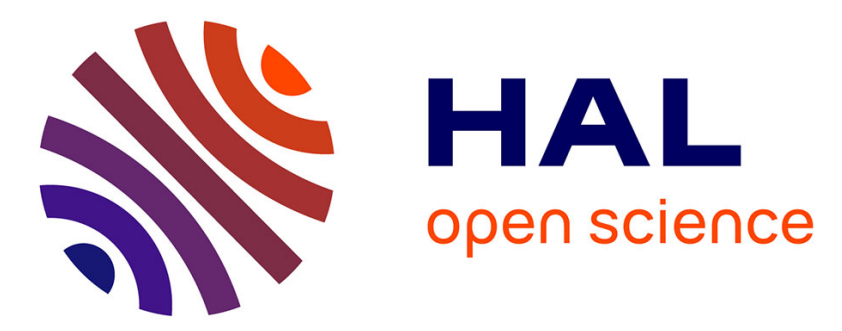

\title{
L'Aménagement des forêts "publiques": point de vue d'un juriste.
}

Jacques Liagre

\section{To cite this version:}

Jacques Liagre. L'Aménagement des forêts "publiques" : point de vue d'un juriste.. Revue forestière française, 1999, 51 (sp), pp.50-59. 10.4267/2042/5504 . hal-03443624

\section{HAL Id: hal-03443624 \\ https://hal.science/hal-03443624}

Submitted on 23 Nov 2021

HAL is a multi-disciplinary open access archive for the deposit and dissemination of scientific research documents, whether they are published or not. The documents may come from teaching and research institutions in France or abroad, or from public or private research centers.
L'archive ouverte pluridisciplinaire HAL, est destinée au dépôt et à la diffusion de documents scientifiques de niveau recherche, publiés ou non, émanant des établissements d'enseignement et de recherche français ou étrangers, des laboratoires publics ou privés. 


\section{L'AMÉNAGEMENT DES FORÊTS "PUBLIQUES" : POINT DE VUE D'UN JURISTE}

\section{J. LIAGRE}

À raison des aspects historiques qui sous-tendent principalement le présent article, nous limiterons son contenu à l'examen de l'aménagement applicable aux forêts bénéficiant du régime forestier. Nous prions les lecteurs de bien vouloir excuser la simplification à laquelle nous avons recours dans le titre pour désigner sous le vocable de "forêts publiques" les forêts ainsi soumises au régime forestier.

Difficile pour un juriste d'oser s'aventurer dans une analyse de l'aménagement forestier. Ne touchet-on pas là au sanctuaire le plus sacré du forestier? Clef de voûte de la gestion des forêts soumises au régime forestier, l'aménagement n'est-il pas d'abord et avant tout domaine réservé du sylviculteur et du gestionnaire technique de l'espace naturel forestier ? Et pourtant...

Pourtant, si l'on veut bien arrêter un instant notre regard sur la face juridique de ce sommet de la technique forestière, quel point de vue superbe on peut obtenir.

- Historiquement tout d'abord, l'aménagement forestier nous apparaît comme un précurseur de l'action administrative contemporaine (p. 51), démarche on ne peut plus moderne amorcée en un siècle où le droit administratif, tout frais sorti de la Révolution de 1789, s'essayait encore à définir les structures et les moyens d'une Administration républicaine.

- Techniquement ensuite - mais ici en termes de technique juridique - l'aménagement forestier ressemble bien à l'outil le plus parfait - ou le moins imparfait - que les pouvoirs publics aient élaboré pour protéger et mettre en valeur l'espace naturel sans le figer pour autant par une réglementation édictée aux seules fins de conservation (p. 55).

- Et ce mérite que nous croyons pouvoir reconnaître en faveur de l'aménagement forestier, c'est à sa nature très particulière qu'il le doit. Mi acte juridique, mi document technique, il allie en une même dynamique la force du droit et la souplesse de l'action. Sans doute est-ce à cause de ce subtil alliage entre les matériaux juridiques et techniques que la doctrine a eu tant de difficultés pour qualifier la nature de l'aménagement au regard des définitions courantes des normes juridiques (p. 53). 


\section{UN PRÉCURSEUR DE L’ACTION ADMINISTRATIVE CONTEMPORAINE}

Aménager une forêt, c'est doter un massif forestier donné d'un ensemble cohérent de décisions qui permettent une gestion rationnelle en fonction d'objectifs divers.

En d'autres termes, l'aménagement forestier, c'est une démarche rationnelle qui cherche à harmoniser les rôles de la forêt, voire à les hiérarchiser selon les circonstances propres à la propriété forestière concernée.

Par une analyse du milieu forestier (topographie, géologie, pédologie, histoire, climat, vulnérabilité à certains phénomènes - incendie - richesse de la faune...), on doit parvenir à fixer des objectifs accessibles en fonction des atouts et des contraintes propres au dit milieu.

Cette démarche, certes, s'est compliquée au fil des décennies. Mais, dès la création du Code forestier de 1827, on en trouvait les lignes de force dans le rapport de présentation devant la Chambre des Pairs qui expliquait qu'on ne pouvait déterminer les règles générales de l'aménagement et de l'exploitation qu'en fonction de "toutes les circonstances (qui) dépendent essentiellement de causes qui varient, pour chaque forêt, suivant la nature du sol, son exposition, la qualité et l'essence des arbres, les besoins particuliers ou publics, la proximité ou l'éloignement des lieux de consommation, la destination des produits, les moyens de transport » (1).

Ainsi, le forestier a-t-il institué, dès 1827, un mode d'intervention lui permettant de tenir compte à la fois de la spécificité d'un espace (caractères économiques, écologiques, climatiques, géologiques, etc.) et des enjeux (production de bois, gestion cynégétique, protection des milieux et des habitats) pour mener à bien son action dans l'intérêt général.

Car, toujours, l'action du forestier dans son rôle d'aménagiste est guidée par cette volonté d'agir " dans le plus grand intérêt de la société ", les bois étant " des objets de première nécessité " qui intéressent "l'ordre public et l'économie politique " pour reprendre autant d'expressions utilisées en 1827 lors de la présentation du projet de code devant les Chambres.

C'est, d'une part, cette diversité des milieux, cette richesse des caractères propres à chaque forêt et, d'autre part, cette pluralité des motifs d'intérêt général (aujourd'hui résumée par la sempiternelle trilogie : production, protection, accueil ; les enjeux se limitant au XIX $\mathrm{X}^{\mathrm{e}}$ siècle aux enjeux de protection et de production) qui ont conduit les pouvoirs publics à organiser une démarche planifiée de l'administration forestière afin de garantir la cohérence de ses actes et l'équilibre de ses interventions.

Démarche d'autant plus moderne et remarquable que nous nous trouvions alors à l'aube de la révolution industrielle, époque où l'initiative privée individuelle s'en venait triomphante, tout droit sortie de la révolution politique de 1789. Bref, époque guère favorable à une intervention globale de l'Administration.

Or, cette idée de disposer d'un plan qui harmonise et équilibre les actions des forestiers en permettant la cohérence des mesures à mettre en œuvre, n'était-ce pas précisément ce que les Pouvoirs publics contemporains s'escriment à réaliser depuis une trentaine d'années ?

C'est ce que le Professeur Jean-Marie Pontier (1997) appelle "la fonctionnalisation du territoire », phénomène qui " a commencé très modestement et très prudemment dans la seconde moitié de ce siècle avec l'idée d'établir des cartes des équipements à réaliser. Cette planification est d'abord apparue dans les domaines où les besoins se sont manifestés avec le plus d'acuité (...) ". Ce qui est recherché, c'est une rationalisation de l'action administrative : "la démarche consiste à recenser les

(1) Rapport du Comte Roy à la Chambre des Pairs, séance du 8 mai 1827 cité par Meaume dans son Commentaire du Code forestier. - Éditions Delamotte et Rimblot, 1844. - Tome 1, p. 71. 
besoins, ces derniers établis (ou jugés tels), à programmer la réalisation des équipements selon une distribution territoriale fixée à partir d'un certain nombre d'indicateurs de besoins. L'objectif est de parvenir à la meilleure adéquation possible entre les besoins et les équipements ».

C'est ainsi que schémas, plans, zonages se multiplient de nos jours. Du plan d'occupation des sols, aux zones d'éducation prioritaires (ZEP) sans oublier les schémas d'aménagement et de gestion des eaux (SAGE) ou les schémas directeurs d'aménagement et de gestion des eaux (SDAGE)... jusqu'à aboutir, par exemple, en 1995 à un regroupement au sein des plans de prévention des risques naturels (PPR) des divers plans élaborés jusqu'alors en matière de sécurité publique (plan d'exposition aux risques, plans des zones sensibles aux incendies de forêt, etc.).

Certes, cette démarche des autorités administratives ne peut être parfaitement assimilée à celle des forestiers. Les unes raisonnent sur des espaces très vastes dépassant les circonscriptions administratives traditionnelles (communes, arrondissements, voire départements) là où les autres raisonnent à l'échelle d'une propriété. Les premières agissent essentiellement en termes de réglementation, d'infrastructures et d'équipements, là où les autres traitent de la gestion dans sa globalité, jusque dans les interventions les plus usuelles. Mais, qu'importent ces différences dès lors que dans les deux processus nous retrouvons cette double volonté :

- d'une part, l'appréhension globale d'un ensemble de données diverses qui constituent autant de paramètres agissant sur l'espace sur lequel on entend intervenir. Qu'importe que le forestier se penche sur les caractères du sol, la flore, le gibier, le climat, tandis que l'administrateur, selon son domaine d'action (santé, scolarité, eau...), se penche sur des données démographiques, sociales, économiques. Dans les deux hypothèses, il s'agit bien d'une même préoccupation : connaître la complexité du système objet de l'intervention.

- d'autre part, la hiérarchisation des objectifs d'intérêt général qui s'attachent à l'action entreprise. Non seulement le milieu est complexe et les paramètres à prendre en compte sont multiples et évolutifs mais encore les buts poursuivis, tout en tendant tous vers l'intérêt général - cette raison d'être de l'action de l'Administration - peuvent rivaliser entre eux.

Là où le forestier doit chercher à valoriser la fonction de production (intérêt général à caractère économique) tout en préservant le milieu naturel (intérêt général à caractère écologique) et en assurant la fonction d'accueil (intérêt général à caractère social mais aussi de plus en plus économique (2)), l'Administration doit pareillement chercher à satisfaire une multitude de besoins divergents, voire contradictoires (3), tels que le développement économique, l'emploi, le maintien d'espaces verts et d'espaces naturels, le transport, le logement, etc.

Le Code forestier de 1827, en instituant l'obligation d'aménager les forêts soumises au régime forestier, a su donner à l'administration forestière un outil remarquablement moderne par son esprit et sa dynamique. Mais, précisément, " le propre de la législation forestière étant de prévoir l'avenir, les législations forestières ont donc cette particularité fondamentale d'intervenir par anticipation, ce qui leur donne un caractère permanent et sans âge " (Casorati, 1998).

$$
* *
$$

(2) II est indiscutable que, depuis quelques années, le tourisme rural et les sports de nature offrent aux petites communes rurales de nouvelles occasions de redynamiser leur économie locale. Ce trait a notamment été souligné dans le Rapport Munet devant le Conseil économique et social : Forêt - espace naturel : de nouvelles missions reconnues pour l'agriculture, fascicule JO $n^{\circ} 4157$. Voir aussi Jacques Liagre. - L'Accueil du public en forêt : fonction sociale de la forêt française. - Économie 1996 - Université de Perpignan, p. 49.

(3) Ainsi, lorsque la loi sur l'eau institue, au travers des Schémas d'Aménagement et de Gestion de l'Eau (SAGE), une volonté de poursuivre simultanément des objectifs que, par le passé, autant de lois distinctes poursuivaient parallèlement, pour ne pas dire en concurrence : intérêt général sanitaire (qualité de l'eau), intérêt général faunistique et floristique (pêche, milieu naturel), intérêt général économique (utilisation de l'eau par les industries). 
Plus que moderne, l'aménagement forestier nous apparaît particulièrement évolutif... c'est-à-dire remarquablement adaptable aux attentes nouvelles de la société et cela sans qu'il soit besoin d'agir sur les textes qui l'ont institué.

Cette souplesse d'adaptation s'explique par la nature mixte du contenu de l'aménagement, mi norme juridique, mi outil technique de gestion, d'où cette difficulté rencontrée par la doctrine pour qualifier la nature exacte de cet acte... Difficulté qu'il nous paraît indispensable d'examiner en premier lieu pour mieux évoquer ensuite l'extraordinaire souplesse de l'aménagement forestier.

\section{ACTE ADMINISTRATIF DE DROIT PUBLIC OU SIMPLE DOCUMENT DE GESTION ?}

Dès l'origine, les Pouvoirs publics ont souligné l'intérêt général qui s'attachait à l'aménagement des forêts. Lors de la présentation du projet de Code forestier, on a ainsi pu affirmer que l'aménagement " est la plus importante partie " de l'Administration des forêts. Et cela parce que l'aménagement est réalisé " dans le plus grand intérêt de la conservation de la forêt, de la consommation générale et (...) dans le plus grand intérêt de la société » (4). On ne saurait être plus clair sur les buts d'intérêt général poursuivis par l'aménagiste.

D'ailleurs, le rapporteur précisait que, si les opérations de délimitation de bornage des forêts " doivent être subordonnées aux principes du droit civil et de la propriété, leur aménagement est soumis à d'autres règles, à celles de la meilleure conservation et à celles d'une sage prévoyance des besoins de l'avenir ».

Si l'aménagement est un outil juridique soumis à des règles autres que celles du droit civil, on doit logiquement en déduire qu'il s'agit d'un acte de droit public, ce droit dont l'intérêt général " est en quelque sorte l'alpha et l'oméga " (Pontier, 1998). Un tel acte, destiné à conserver et protéger les peuplements revêtant dès lors une force juridique exorbitante qui en fait une décision administrative exécutoire ${ }^{(5)}$.

Or, de façon tout à fait surprenante, les Pouvoirs publics et la doctrine se sont refusés à pousser jusqu'au bout ce raisonnement. Longtemps l'administration forestière a écarté l'idée que l'aménagement forestier puisse être un acte administratif à part entière, constitutif d'une véritable décision. Apparaît ainsi une contradiction entre l'usage qu'on entend faire de l'aménagement forestier et la définition qu'on en donne.

Le vicomte de Martignac, ministre d'État chargé des Forêts, ouvre lui-même la route à cette contradiction en expliquant que tout ce qui touche au mode de régie des bois de l'État, à la police intérieure de leur administration, à leur aménagement relève d'une " "administration intérieure des forêts" (au sens de gestion) qui incombe au Gouvernement et non au législateur ", ainsi la loi " ne règle pas cet aménagement, parce que ce règlement est un acte matériel d'administration qui n'est pas du domaine de la loi »(6). Discours encore renforcé dans le rapport effectué devant les députés : “(l'aménagement) est incontestablement un acte d'administration » (7).

Le ton était donné. Nul n'osera plus remettre en cause cette analyse durant plus d'un siècle.

\footnotetext{
(4) Présentation du projet de Code forestier. Rapport du Comte Roy, séance du 8 mai 1827 devant la Chambre des Pairs. Meaume, op. déjà cité, p. 70.

(5) «Une décision exécutoire est un acte juridique émis unilatéralement par l'administration en vue de modifier l'ordonnancement juridique par les obligations qu'il impose ou par les droits qu'il confère ". Georges Vedel. - Droit administratif. - Éditions Thémis, 1976, p. 180.

(6) Exposé des motifs devant la Chambre des Députés, 29 décembre 1826. Meaume, op. déjà cité, p. 4 et 7.

(7) Rapport du Baron Favard de Langlade à la séance du 12 mars 1827 devant les Députés. Meaume, op. déjà cité.
} 
En 1910, Charles Guyot écrivait dans son cours de droit forestier : «les décrets d'aménagement ne sont soumis à aucune publicité, ils ne sont susceptibles d'aucun recours ". Et, en 1968, soit un siècle et demi après la promulgation du Code de 1827, l'ingénieur général Meyer reprenait après Guyot : "Ces arrêtés (d'aménagement) ne sont pas publiés au Journal Officiel et ne sont pas susceptibles de recours de la part des tiers. Ce sont des actes de gestion internes à l'Administration ».

L'aménagement forestier, même s'il est plus qu'un simple acte de gestion privé, ne serait donc, selon la doctrine et pour reprendre un concept issu de la jurisprudence du Conseil d'État relative aux instructions, directives et autres circulaires, qu'un " acte d'administration intérieure " (8) dénué de véritable force juridique et seulement utile « à la vie intérieure » de l'Administration.

Cette définition nous semble pourtant trop audacieuse pour résister à une analyse plus serrée : comment l'aménagement pourrait-il n'être qu'une mesure d'ordre administratif interne au service public forestier, comme le sont les instructions et circulaires alors même que de nombreuses dispositions législatives et réglementaires viennent affirmer la force juridique exorbitante qui s'y attache?

D'une part, l'un des objets majeurs de l'aménagement, la planification des coupes, constitue un programme à caractère obligatoire dont toute dérogation implique l'obtention d'une autorisation administrative expresse et préalable (art. 16 CF de 1827, L. 133.2 actuel). Cette force juridique qui s'attache au programme des coupes fixé dans l'aménagement est si vraie que :

- toute vente portant sur une coupe non réglée à l'aménagement ou non autorisée par l'autorité administrative est frappée de nullité (art. L. 133.2 CF) ;

- l'état d'assiette qui permet de contrôler le respect de l'aménagement est un acte détachable de la gestion du domaine privé qui constitue un acte administratif relevant d'une mission de service public administratif (conservation et protection de la forêt) et, à ce titre, est susceptible de recours de la part de tiers ${ }^{(9)}$;

- le législateur a estimé qu'un terrain boisé soumis au régime forestier donc bénéficiant d'un aménagement forestier échappe au régime d'autorisation administrative de coupes instauré par l'article L. 130.1 du code de l'Urbanisme.

D'autre part, tout projet de travaux ou d'occupation du sol forestier est soumis au contrôle préalable pour s'assurer de sa compatibilité avec l'aménagement. Cette opération qui se déduisait anciennement de l'article L. 143.2 CF est aujourd'hui exprimée d'une manière autrement plus évidente par la nouvelle rédaction de l'article R. 143.2 CF (10).

Tout cela constitue autant d'éléments objectifs, qui confèrent à l'aménagement forestier une force juridique bien supérieure à celle qui s'attache à un simple acte d'administration intérieure.

Voici donc contredite l'idée que la doctrine entretenait depuis plus d'un siècle et demi. Comment expliquer cette vision juridiquement restrictive que la doctrine a donnée de la nature de l'aménagement forestier durant plus d'un siècle?

(8) CE 22 février 1918 Cochet d'Hattecourt rec. p. 176. Note Hauriou S. 1921.3.9.

(9) «Considérant que si les contestations auxquelles donne lieu la gestion des forêts de l'État par l'Office national des Forêts relèvent en principe des tribunaux de l'Ordre judiciaire en raison de l'appartenance de ces forêts au domaine privé et, au surplus, du caractère industriel et commercial dudit Office, la décision par laquelle le Ministre de l'Agriculture et, le cas échéant, les représentants de l'Office national des Forêts, agissant par délégation du Ministre, autorisent conformément au Code forestier et à l'ordonnance réglementaire du 1er août 1827, l'assiette des coupes de bois dans les dites forêts, se rattache à l'exécution d'une mission de service public de protection de la forêt, présentant un caractère administratif et constitue un acte administratif détachable de la gestion du domaine privé ". CE, 3 mars 1975, Courrières et autres rec. p. 165 et AJDA, $1975, n^{\circ} 48$ note Boyon et Franc, p. 233.

(10) $2^{\circ}$ alinéa de l'article R. 143.2 nouveau du Code forestier issu de l'article 2 du décret n 97.1163 du 17 décembre 1997 $2^{\circ}$ alinéa : "Le représentant de la collectivité ou personne morale propriétaire consulte l'Office national des Forêts sur la compatibilité, avec l'aménagement arrêté, des projets de travaux ou d'occupation concernant des terrains soumis au régime forestier ». 
Bien des motifs sous-tendent cette situation :

- Tout d'abord, la doctrine ne s'est jamais interrogée que sur l'aménagement des forêts domaniales. C'est-à-dire une hypothèse où l'État joue de manière ambiguë un rôle à la fois de Puissance publique et de propriétaire.

- Ensuite, s'agissant des forêts des collectivités, il faut se rappeler qu'à l'époque les communes étaient sous une forte tutelle de l'État. L'idée que celui-ci puisse arrêter leur aménagement ne provoquait donc pas forcément d'interrogation sur la nature exorbitante de ce régime. De surcroît, le Code forestier ne comportait alors aucune disposition traitant de l'aménagement des forêts des collectivités et se contentait de renvoyer au texte (art. 15) relatif à l'aménagement des forêts domaniales. L'amalgame n'en était que plus aisé.

- La difficulté résidait aussi dans la définition même de l'acte en cause. Comment qualifier l'aménagement ? Ce n'est pas un contrat puisqu'il est réglé unilatéralement par l'autorité publique. Ce n'est pas davantage un acte réglementaire puisqu'un tel acte a, par définition, un caractère général et impersonnel, or, l'aménagement ne s'applique que sur une seule propriété forestière et n'avait, jusqu'à une date récente, aucun effet contre les tiers.

Quant à la décision individuelle, celle-ci doit normalement être notifiée à l'administré auquel elle s'applique. Or, rien de tel n'était prévu dans les textes. De surcroît, le Code de 1827 renvoyant, pour ce qui concerne l'aménagement des forêts des collectivités, aux dispositions applicables à la forêt domaniale, force était d'admettre à l'époque que, dans l'esprit même du législateur, l'aménagement tenait plus de l'instruction interne que de la décision individuelle.

L'examen de l'Ordonnance royale montre que celle-ci ne donnait que des prescriptions techniques, ce qui rendait peu évident son éventuel caractère normatif (11).

À défaut d'apparence "décisionnelle" et de toute procédure prévoyant une publication ou une notification, l'aménagement revêtait bien une apparence d'acte d'administration interne et ce à une époque où les tribunaux, alors plus soucieux de défendre l'efficacité de l'action administrative que les droits et libertés des citoyens, n'hésitaient pas à invoquer fréquemment ce concept de mesures d'ordre intérieur pour s'autocensurer dans leur contrôle de l'administration (12) .

$$
* *
$$

Et, peut-être, cette vision réductrice de la nature juridique de l'aménagement forestier est-elle à l'origine de cette remarquable faculté d'adaptation qui permet à ce document de remplir en 1999 un rôle d'une extrême complexité, ô combien différent et plus complet que celui pour lequel il avait été institué en 1827. Et cela quasiment sans avoir nécessité la moindre modification dans la rédaction des textes législatifs et réglementaires qui le fondent.

\section{L'AMÉNAGEMENT FORESTIER, UN REMARQUABLE OUTIL ÉVOLUTIF}

Si l'on s'arrête sur la définition du contenu de l'aménagement, telle que la donnait la doctrine au début du XIX ${ }^{e}$ siècle, on y découvre des préoccupations essentiellement techniques et économiques limitées à la seule exploitation des coupes.

(11) Article 68 de l'Ordonnance royale du $1^{\mathrm{er}}$ août 1827 : « Les aménagements seront réglés principalement dans l'intérêt des produits en matière et de l'éducation des futaies. En conséquence, l'administration recherchera les forêts et parties de forêts qui pourront être réservées pour croître la futaie. Elle en proposera l'aménagement en indiquant celles où le mode d'exploitation par éclaircie pourrait être le plus avantageusement employé ". Article 69 : "Dans toutes les forêts qui seront aménagées à l'avenir, l'âge de la coupe de taillis sera fixé à 25 ans au moins et il n'y aura d'exception à cette règle que pour les forêts dont les essences dominantes seront le châtaignier et les bois blancs ou qui seront situées sur des terrains de la dernière qualité "... Voilà qui ressemble bien plus à un guide technique de sylviculture qu'à un acte réglementant les conditions d'exercice du droit de propriété. (12) La situation a considérablement évolué depuis en matière d'acte d'administration interne. "Actuellement l'état du droit en ce qui les concerne se trouve profondément modifié par une évolution jurisprudentielle restreignant au maximum cette catégorie de mesures ». B. Chapus. - Droit administratif général. Tome I. - 8 édition. - Édition Montchrestien, $1995 .-$ p. $454 \S \mathrm{n}^{\circ} 565$. 


\section{J. LIAGRE}

Le Code forestier commenté Dalloz de 1884 rappelait fort à propos dans ses commentaires de l'article 15 que "l'aménagement des forêts ne s'entendait autrefois que des soins et économies apportés dans l'exploitation. Cette acception était encore reçue vers le milieu du XVIIIe siècle (...) ».

En 1827, l'aménagement est défini comme "l'art de diviser une forêt en coupes successives et de régler l'étendue et l'âge des coupes annuelles dans le plus grand intérêt de la conservation de la forêt, de la consommation en général, dans celui enfin du propriétaire et s'il s'agit de forêts de l'État dans le plus grand intérêt de la société ".

Autant de définitions qui, même axées sur un souci de conservation des peuplements, demeuraient liées à la fonction économique de la forêt (récolte des bois).

Lorsqu'en 1884, le Code forestier commenté Dalloz définit l'aménagement comme " une opération qui consiste à régler le mode de culture de la forêt ainsi que la marche et la qualité des exploitations de manière à assurer annuellement une succession constante et égale des meilleurs produits possibles ", il faisait certes évoluer la précision de l'outil technique sylvicole, mais, sans sortir de la seule préoccupation économique de l'exploitation des coupes. De plus, juridiquement, on demeurait dans une conception exclusivement civiliste de l'aménagement.

En effet, il est intéressant de faire observer que le Code civil se réfère de son côté à un concept d'aménagement à propos de l'exploitation des coupes dans les forêts grevées d'un usufruit.

L'aménagement, au sens du Code civil (article 590 et suivants Code civil), peut se définir comme une pratique habituelle qui revêt la force d'un usage. En d'autres termes, on peut dire qu'en droit civil, l'aménagement d'un bois ou d'une forêt est l'expression d'une volonté manifeste des propriétaires de se créer une source de revenus régulière et périodique. II ne suffit pas qu'il y ait annualité des coupes : celles-ci doivent, par leur nature, démontrer qu'il existe une gestion organisée de la propriété forestière ${ }^{(13)}$.

II y a ici une parfaite harmonie entre l'aménagement d'une forêt usufruitière (Code civil) et la définition de l'aménagement d'une forêt soumise au régime forestier donnée dans le Code forestier commenté Dalloz de 1884. Dans les deux cas, aménager la forêt revient à rechercher, dans une préoccupation de rendement soutenu, des coupes dont la périodicité et la régularité dénotent une gestion organisée ; les prélèvements ne préjudicient donc en rien au capital, n'altérant pas la substance du fonds et garantissant une rotation harmonieuse des coupes dans l'avenir (14).

Nous voici au cœur d'une vision privatiste et purement patrimoniale de la gestion forestière.

Une évolution sensible s'amorce, en 1910, avec Charles Guyot qui présente l'aménagement comme " une règle de conduite qui trace le devoir du gestionnaire pour conserver les forêts dans leur intégralité et les améliorer autant que possible notamment en déterminant les revenus et en les réalisant de la manière la plus avantageuse pour la forêt ". Cette idée " d'améliorer autant que possible » les forêts nous éloigne sensiblement de l'art de diviser une forêt en coupes successives et d'en régler l'étendue et l'âge.

Sautons encore quelques décennies. L'ingénieur général Meyer nous fait plonger en 1968 dans une vision autrement plus large du contenu de l'aménagement.

Celui-ci est devenu « une opération qui fixe les objectifs de gestion pour une durée déterminée en tenant compte de critères de rentabilité et d'intérêt général. L'aménagement doit assurer la production la mieux adaptée aux besoins de l'économie locale et nationale, maintenir l'équilibre naturel de la forêt et assurer le renouvellement régulier des peuplements pour ne pas compromettre l'utilité

(13) Voir notamment arrêt de la Cour de Cassation, 14 mars 1838 - D 1838-1-140.

(14) Définition qu'on retrouve parfaitement dans un arrêt de la Cour d'Appel de Paris du 10 octobre 1959 - D $1959-2-264$. 
générale du massif ; enfin, prévoir le programme des travaux d'entretien, d'amélioration, de protection et d'équipement ".

L'intérêt général y est enfin annoncé comme un des ressorts de l'action de l'aménagiste. À la planification des coupes, s'ajoute la programmation des travaux et surtout, à l'enjeu économique, vient s'ajouter la préoccupation écologique du maintien de l'équilibre naturel de la forêt... Que de chemin parcouru depuis 1827.

Quelle évolution de la démarche de l'aménagiste, qui nous permet de disposer désormais d'un document complet, donnant une vision globale des potentiels et des enjeux, et d'harmoniser les actions à entreprendre.

Or, cette évolution du contenu de l'aménagement ne s'est accompagnée d'aucune modification législative ou réglementaire. Le Code forestier dans son édition de 1970 se réfère toujours à la seule formule de 1827 seulement transformée par la déconcentration des pouvoirs de l'État.

Dans sa rédaction de 1827, l'article 15 se contentait d'affirmer : " tous les bois et forêts du domaine de l'État sont assujettis à un aménagement réglé par des ordonnances royales " (15). La même formule se retrouve plus d'un siècle plus tard: " tous les bois et forêts du domaine de l'État sont assujettis à un aménagement réglé par des arrêtés du ministre de l'Agriculture ". Formule qui deviendra "par arrêté ministériel » lors de la codification moderne opérée en 1979 (nouvel art. L. 133.1). Cette recodification est d'ailleurs l'occasion d'introduire dans les textes la procédure d'aménagement des forêts des collectivités (16).

En d'autres termes, le législateur n'est pas intervenu une seule fois pour modifier sur le fond l'article 15 du Code forestier entre 1827 et 1979, alors même que, dans les faits, la démarche du forestier aménagiste avait considérablement évolué dans le même laps de temps.

Certes, le décret $n^{\circ} 77.1141$ du 12 octobre 1977 pris pour l'application de la loi du 10 juillet 1976 sur la protection de la nature est venu poser le principe suivant lequel les aménagements forestiers devront inclure des préoccupations d'environnement. Mais cette disposition n'est pas intégrée dans le Code forestier. Cela nous conforte dans l'idée que l'aménagement peut s'enrichir de préoccupations nouvelles, dues notamment à l'émergence de législations particulières dans d'autres domaines, notamment la protection de l'environnement, sans qu'il soit besoin de modifier pour autant le Code forestier.

De même, lorsque, dans les années 1980, le ministre de l'Environnement, le ministre de l'Agriculture et l'Office national des Forêts conviennent de favoriser la création de réserves biologiques dans les forêts soumises, l'aménagement forestier va permettre la création de ces réserves par une simple adaptation de la démarche de l'aménagiste sans aucun recours à une quelconque disposition réglementaire spécifique.

Ce n'est qu'en 1991, que le Code forestier connaît, pour la première fois depuis 1827, une modification importante relative au contenu même de l'aménagement. Un second alinéa nouveau est introduit dans l'article L. 133-1 (ancien article 15) qui dispose que "l'arrêté d'aménagement peut, pour certaines zones, interdire ou soumettre à des conditions particulières les activités susceptibles de compromettre la réalisation des objectifs de l'aménagement ". Disposition législative qui aura pour corollaire un article R. 133.1.1 nouveau déterminant les modalités de création de ces zones réglementées et instituant une contravention de $4^{\mathrm{e}}$ classe à l'encontre de ceux qui y contreviennent.

(15) On a vu que, s'agissant des forêts communales, il n'existait pas de dispositions spécifiques aux aménagements des forêts des collectivités, le $\S 3$ de l'article 90 se contentant de rendre applicables aux forêts soumises non domaniales les dispositions de l'article 15.

(16) Art. L. 143.1 CF : "Les aménagements des bois et forêts du domaine des collectivités et personnes morales mentionnées à l'article L. 141.1 sont réglés par des arrêtés ministériels conformément aux dispositions des articles L. 133.1 et L. 141.2 ". 
En réalité, cette modification législative n'invente rien. Loin d'imposer à l'aménagiste une démarche nouvelle, la loi ne fait que prendre en compte une dynamique déjà instaurée de facto sur le terrain. Le seul objet de cette modification législative était de donner aux mesures de protection des milieux instaurées spontanément par les aménagements (notamment les réserves biologiques créées depuis 1980) une réelle force juridique les rendant opposables aux tiers.

Techniquement, il n'était nul besoin d'une modification du Code forestier pour intégrer dans l'aménagement les démarches de protection des milieux naturels. Depuis des années, l'aménagiste agissait spontanément selon les spécificités des milieux en cause et les orientations retenues localement. Posait problème la seule question de l'efficacité des mesures adoptées par le gestionnaire : faute d'un dispositif réglementaire les rendant opposables aux tiers et prévoyant une sanction en cas de violation, le forestier ne pouvait guère imposer leur respect aux divers ayants droit et autres publics utilisateurs de la forêt.

Cette modification législative, opérée en 1991, est donc particulièrement originale puisque le législateur, loin d'intervenir pour insuffler à l'Administration une dynamique ou lui imposer une procédure nouvelle, comme c'est son rôle habituel, a eu pour seule motivation effective de prendre en compte l'action spontanée des gestionnaires en consacrant dans la loi ces mesures d'intervention.

En d'autres termes, c'est à partir de l'aménagement forestier tel qu'il était institué par le Code de 1827 que les forestiers ont pu mettre au point, dans les années 1980-1990, un système de zonage et de réserves biologiques permettant de protéger le milieu naturel. L'intervention a posteriori du législateur n'a eu d'autres buts que de consacrer cette démarche de protection en lui donnant la force juridique nécessaire pour en garantir l'efficacité.

Un tel phénomène mériterait bien d'autres développements. II serait notamment très intéressant de se livrer à une étude comparée entre l'aménagement forestier, tel qu'il se trouve défini dans ses objectifs et son contenu par l'actuel article L. 133.1 du Code forestier, et les autres mesures juridiques instituées par les lois et règlements en matière de gestion et de protection des espaces naturels. Tel n'est pas l'objet du présent article qui se voulait, avant tout, être un éclairage juridique et historique sur l'évolution du concept d'aménagement forestier.

En une époque où tant d'hommes politiques se plaignent de l'inflation des textes législatifs et réglementaires, l'aménagement forestier vient démontrer qu'un outil technique et juridique, vieux de près de deux siècles, peut parfaitement permettre, avec souplesse et efficacité, sans intervention du législateur, l'adaptation des interventions des gestionnaires de milieux naturels aux exigences de la société contemporaine.

Le législateur a bien compris l'opportunité et l'intérêt d'un tel mode de planification de la gestion forestière, qui a considéré que l'existence d'un aménagement valait bien mieux qu'une réglementation rigide strictement crispée sur des préoccupations de protection. Ainsi l'article L. 130.1 du code de l'Urbanisme, destiné à protéger les espaces boisés classés à conserver aux plans d'occupation des sols, écarte expressément le régime d'autorisation administrative des coupes lorsque le terrain en cause est doté d'un aménagement forestier ou d'un plan simple de gestion.

Ce "régime de faveur" institué par le code de l'Urbanisme dans une matière particulièrement récente ${ }^{(17)}$ montre qu'il est possible aux Pouvoirs publics de conjuguer avec cohérence des régimes juridiques traitant de matières différentes et qu'ainsi une mesure instaurée en 1827 pour des motifs alors prioritairement économiques peut parfaitement satisfaire aux exigences d'une législation moderne répondant à des préoccupations d'ordre essentiellement paysager. Ce remarquable mariage entre le code de l'Urbanisme et le Code forestier nous confirme que "l'aménagement

(17) Le régime des espaces boisés classés, d'abord institué en 1958 par un décret qui fut annulé par le Conseil d’État, a été régulièrement instauré par la loi n²7.626 du 10 juillet 1973. 
forestier est une démarche exemplaire qui trouve ses racines dans des siècles d'histoire forestière tout en s'adaptant aux exigences et réalités d'un monde moderne avide de protection écologique " (Le Meur, 1998).

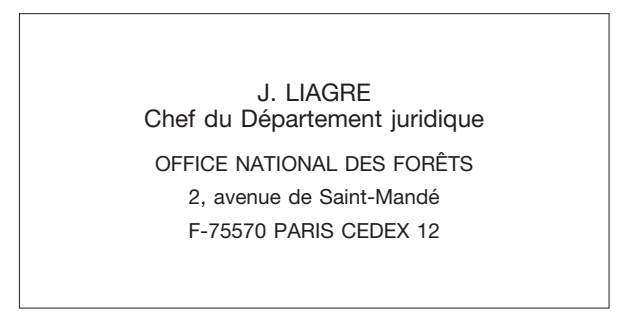

\section{BIBLIOGRAPHIE}

CASORATI (J.). - Le Droit forestier, l'homme et la forêt. - Arborescences, $\mathrm{n}^{\circ}$ 74, mai-juin 1998, pp. 2-3. GUYOT (Ch.). - Cours de Droit forestier. - Paris : Éd. Lucien Laveur, 1908. - Tome 2, § 1210, pp. 367.

LE MEUR (S.). - L'Aménagement forestier, un outil de gestion séculaire au service de la protection de l'environnement. - Arborescences, ${ }^{\circ} 74$, mai-juin 1998, pp. 4-6.

MEAUME (E.). - Commentaire du Code forestier. - Paris : Éd. Delamotte ; Nancy : Grimblot, 1844. - Tome 1, pp. 1-95.

MEYER (F.). - Législation et politique forestières. - Paris : Éd. Berger-Levrault, 1968. - 300 p. (p. 54).

PONTIER (-). - Territorialisation et déterritorialisation de l'action publique. - AJDA, octobre 1997, p. 723.

PONTIER (-). - L'Intérêt général existe-t-il encore ? - Paris : Dalloz, 1998. - Chronique, p. 327.

\section{L’AMÉNAGEMENT DES FORÊTS “PUBLIQUES” : POINT DE VUE D’UN JURISTE (Résumé)}

Précurseur de l'action administrative, l'aménagement forestier apparaît en effet comme un document qui harmonise et équilibre les actions du forestier, ce qu'on retrouve aujourd'hui dans tant d'autres démarches administratives tels par exemple les plans d'occupation des sols, les plans de prévention des risques, etc. où, à chaque fois, l'autorité administrative cherche à satisfaire une multitude de besoins divergents, voire contradictoires. Ainsi, le législateur de 1827 a-t-il créé avec l'aménagement forestier un outil remarquablement moderne par son esprit et sa dynamique.

Mais plus encore que moderne, l'aménagement apparaît extraordinairement évolutif, s'adaptant aux attentes nouvelles de la société sans même qu'il ait été besoin de modifier la loi initiale.

Cette faculté d'adaptation, quasi spontanée, c'est à sa nature très originale qu'il la doit. Mi acte juridique, mi document technique de gestion, il allie en une même dynamique la force du droit et la souplesse de l'action, ce qui explique peut-être la difficulté qu'on a à définir sa nature exacte au regard des définitions courantes des normes juridiques.

\section{PUBLIC FOREST PLANNING - A LEGAL PERSPECTIVE (Abstract)}

A precursor in the area of administrative action, the forest development plan may be viewed as a document that harmonises and balances foresters' interventions, just as is the case with a variety of other administrative measures such as the land use plans, hazard prevention plans, etc. In each of these cases, the administrative authority attempts to meet a range of diverse, sometimes contradictory, needs. By providing for the forest development plan, the lawmaker in 1827 established a tool whose spirit and dynamics are remarkably modern.

Moreover, the forest development plan is extraordinarily adaptable and has adjusted to the new demands of society without even needing amendments of the original law.

It owes this almost automatic adaptability to its very original character. As a hybrid between a legal act and a technical management document, it combines the strength of law and the flexibility of action within a single dynamic, which perhaps explains why it is so difficult to define its exact nature in common legal terms. 